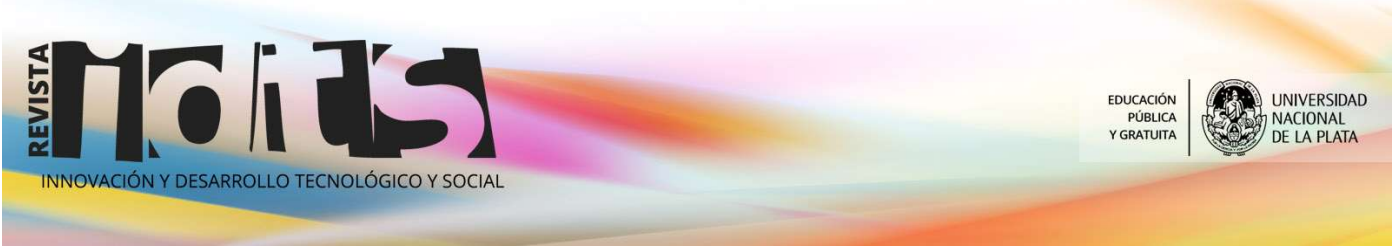

Innovación y Desarrollo Tecnológico y Social (2019) 1 (2): 28-35

\title{
Sistema de software para la gestión energética enmarcado en la norma IRAM-ISO 50001
}

\author{
Bifano, Lautaro ${ }^{1,3}$; Maccarone, José ${ }^{2}$ \\ ${ }^{1}$ Codiseño Aplicado, CODAPLI UTN FRLP \\ ${ }^{2}$ Grupo de Gestión y Tecnología Energética, GyTE UTN FRLP \\ 3bifanolautaro@frlp.utn.edu.ar
}

Resumen Para que las Pequeñas y Medianas Empresas (PyMEs) no sean obligadas a traspasar todos los incrementos energéticos a precio es necesario aplicar herramientas de gestión enfocadas a mejorar el desempeño energético del conjunto, procurando un resultado de menor consumo a igual producción o igual consumo con mayor producción. El objetivo del presente proyecto es desarrollar un sistema soporte en software en línea para la implementación, seguimiento y control en apoyo a las PyMEs para agilizar el camino en post de objetivos de un uso más racional de la energía, es decir lograr una mayor eficiencia en el consumo energético de las empresas. El software es desarrollado y está siendo mantenido con metodología ágil con modelo de espiral.

La metodología para el desarrollo de la dinámica del software se toma de desarrollos propios y basados en el sistema de gestión enmarcado en la norma IRAM 50001-2018, mientras que los datos para las pruebas se toman de la experiencia adquirida a través de la participación del grupo GyTE (Grupo de Gestión y Tecnología Energética de la UTN FRLP) en análisis energético de procesos productivos y basados en resultados y soluciones aplicadas en empresas PyMEs de la Región. Se muestran los resultados de avance del software a la fecha utilizando los datos reales de una Organización.

Concluimos que este software es una herramienta sencilla de utilizar para ayudar a la implementación de un Sistema de Gestión Energética, disponible online a través del sitio web https://sge.frlp.utn.edu.ar/ y en condiciones de ampliación y/o adaptación para la toma de datos de medición en forma online.

Palabras claves: PyMEs; Pequeñas y Medianas Empresas; SGE; Sistema de Gestión Energética; programa; energía; industria.

Recibido 01/07/2019 Aceptado 09/11/2019

DOI $10.24215 / 26838559 \mathrm{e} 008$

\section{SOFTWARE SYSTEM FOR ENERGY MANAGEMENT FRAMING ON NORM IRAM-ISO 500001}

\footnotetext{
Abstract In order not to transfer the energy increases to prices, it is necessary for Small and Medium Companies (PyMEs) to use management tools focused on improving the whole energy development, trying to obtain a result of a lower consumption at the same level of production or an even consumption at a higher level of production. The aim of the current project is to develop a supporting system in software on line, in accordance with the implementation, follow-up and control to support PyMEs. Then, the way to obtain the desired objectives will be accelerated and the use of energy will be more rational; i.e. obtaining more efficiency in the use of energy by companies. The software was developed and is still being kept in an agile spiral model.

The method of developing the dynamic of the software is taken from our own developments, based on the management system framed in the IRAM 50001-2018 norm; while the information for tests is obtained from experiences acquired by the group GyTE (Management and Energy Technology of UTNFRLP) in the
} 


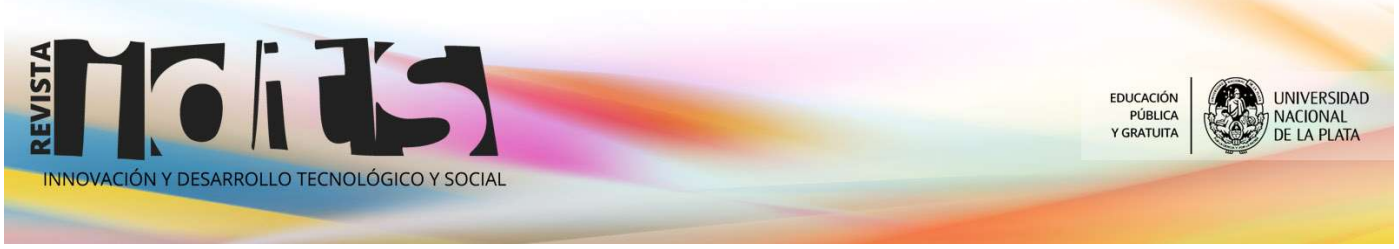

Innovación y Desarrollo Tecnológico y Social (2019) 1 (2): 28-35

energy analysis of productive processes and results used in PyMEs companies in the region. The current results of progress of the software are shown using real data of an organization.

Finally, this software is a simple tool to help the implementation of a System of Energy Management, available on line https://sge.frlp.utn.edu.arl. Besides, it can be further developed and/or adapted to obtain data for measure on line.

Keywords: Small and Medium Companies; SEG; System of Energy Management; Software; industry.

\section{Novedad u originalidad local en el conocimiento}

Para evitar que las PyMEs deban traspasar todos los incrementos energéticos a precio es necesario aplicar herramientas de gestión enfocadas a mejorar el desempeño energético del conjunto, procurando un resultado de menor consumo a igual producción o igual consumo con mayor producción.

En la actualidad, la mayoría de las empresas y organizaciones orientadas a la producción hacen un uso inadecuado de la energía, utilizando y derrochan más de la necesaria. Analizamos la gestión de la demanda y el uso de la energía, con la finalidad de determinar características similares y desde esa base, elaborar la matriz energética típica del sector innovando en el desarrollo de una herramienta de software para la gestión de los energéticos demandados y utilizados por las PyMEs.

\section{Grado de relevancia}

La energía es un insumo para las empresas, con igual precio para todas ya que se adquiere a un mismo valor y su precio no puede negociarse. Su sustitución termina siendo generalmente más cara, por lo tanto, quedan dos caminos, transferir el gasto directamente a los costos del producto o servicio, o gestionar su consumo y su uso. En esta última forma es que este insumo se convierte en una variable competitiva, a través de "La Gestión Energética".

Con el fin de adecuar los usos energéticos de las PyMEs a las recomendaciones de la Norma IRAM-ISO 50001, el sistema objeto de este trabajo promueve políticas que llevan a las empresas a reducir el consumo de energía, de manera que las organizaciones se adapten a la Norma y a la vez cumplan objetivos medioambientales.

\section{Grado de pertinencia}

Teniendo en cuenta las especificaciones y recomendaciones de la Norma IRAM-ISO 50001, se puede resolver el problema energético anteriormente expuesto de las empresas.

A partir de la implementación de este proyecto se van a poder tomar decisiones acerca de cómo proceder para hacer más eficiente el consumo energético en una empresa. En todo momento se pueden obtener reportes e informes para sacar conclusiones respecto de los parámetros energéticos tenidos en cuenta y tomar decisiones a tiempo. El sistema también está provisto de la gestión de No Conformidades y Planes de Acción para, a través de estos, realizarse los cambios correspondientes para cumplir con los objetivos energéticos.

Este proyecto también apunta a concientizar a la sociedad sobre el derroche de energía y a recuperarnos de la crisis energética en la que estamos sumidos en Argentina. 


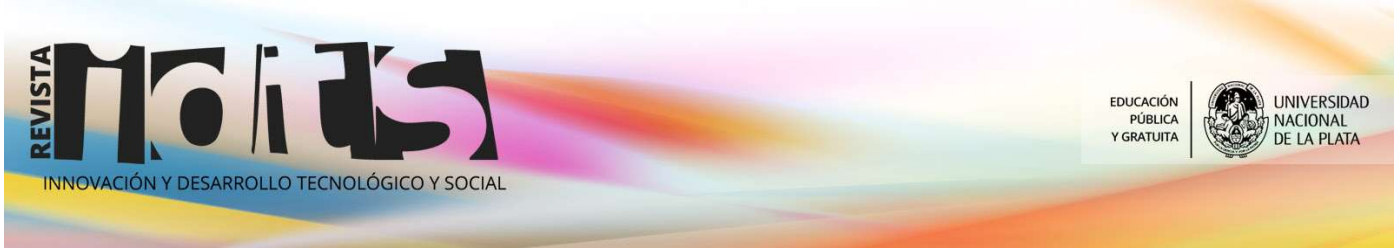

Innovación y Desarrollo Tecnológico y Social (2019) 1 (2): 28-35

\section{Grado de demanda}

En el Congreso Internacional de Distribución Eléctrica (CIDEL) desarrollado en el año 2014, el grupo RedTecNEE presentó un proyecto para analizar la gestión de la demanda y el uso de la energía que realizan las PyMEs de los distintos sectores, con la finalidad de determinar características similares y desde esa base, elaborar la matriz energética típica del sector para desarrollar una herramienta software para la gestión de los energéticos demandados y utilizados por las empresas (RedTecNEE 2014).

Este proyecto fue impulsado y desarrollado en el año 2018 por el grupo Grupo de Gestión y Tecnología Energética (GyTE) y el Laboratorio CODAPLI, sin tener ningún agente adoptante que financie el proyecto. Este se desarrolló con la expectativa de prestar servicio a las PyMES, y que el mantenimiento del sistema sea financiado por estas empresas.

\section{Desarrollo del producto}

Este desarrollo responde al proyecto "RED TECNOLOGICA NACIONAL SOBRE EFICIENCIA ENERGÉTICA" (RedTecNEE 2014) cuyo objetivo general es proveer a las distintas organizaciones de los diferentes sectores, de una herramienta capaz de ayudarles en la gestión y la eficiencia energética mejorando así su nivel de competitividad y por su parte contempla los requisitos para en el futuro poder implementar y/o certificar la Norma IRAM-ISO 50001 de Gestión de la Energía (en adelante la Norma).

El proyecto aborda como base la Norma y sus guías de referencia para la Gestión de la Energía en las Organizaciones, siendo las mismas de alcance nacional e internacional.

Para explicar el desarrollo del Sistema para implementación, seguimiento y control de un Sistema de Gestión Energética (SGE) basado en la Norma, se relaciona las acciones reales de campo con el contenido del Software en desarrollo (en adelante SGEfrlp).

Partiendo de una Revisión Inicial sobre los Consumos de Energía de la PyME en estudio se puede obtener una relación de Energías en sus unidades originales o llevadas a una misma unidad, en este caso serán comparables y se muestra a través de una Matriz de Consumo Energético o a través de un Gráfico tipo Torta [Figura 1].

El SGEfrlp admite que se puedan cargar las facturas de todos los tipos de energía que consume la empresa en sus procesos productivos. De las facturas se especifica el periodo al cual pertenecen, el consumo en la unidad de medida que corresponda según el tipo de energía, el monto que se pagó por esta y datos particulares de cada tipo de energía.

Con la información de todas las facturas se realiza una estadística del consumo energético de la empresa, como se muestra en la siguiente Figura 2. 


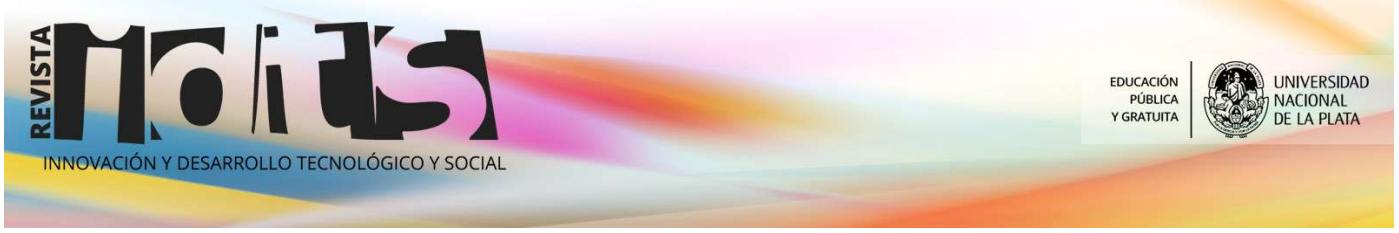

Innovación y Desarrollo Tecnológico y Social (2019) 1 (2): 28-35

\section{Consumo por energía (\% kWh)}

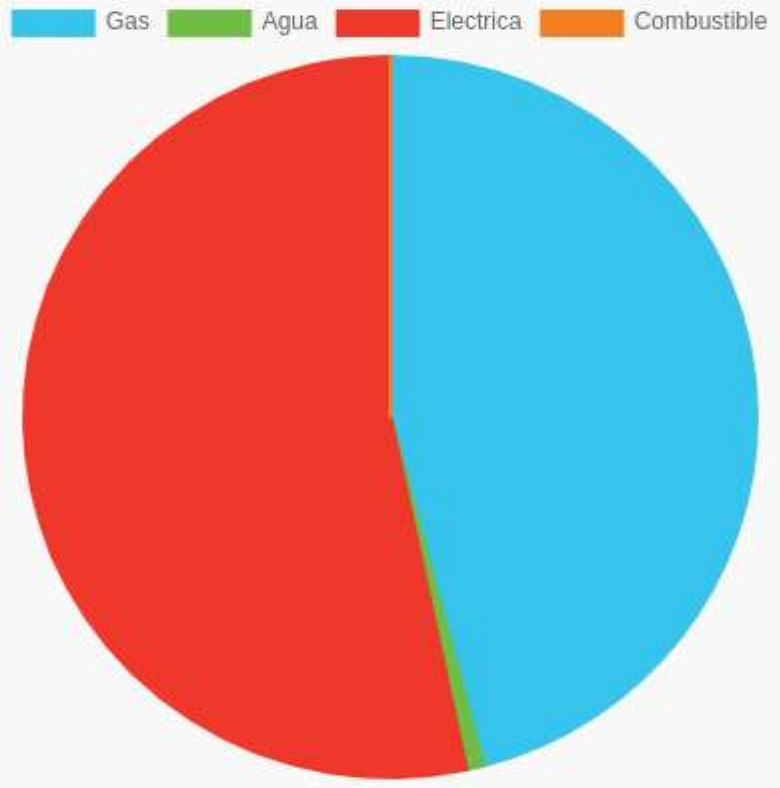

Figura 1: Matriz de Consumos Energéticos Figure 1: Energy consumption matrix

\section{Consumo por energía (kWh)}

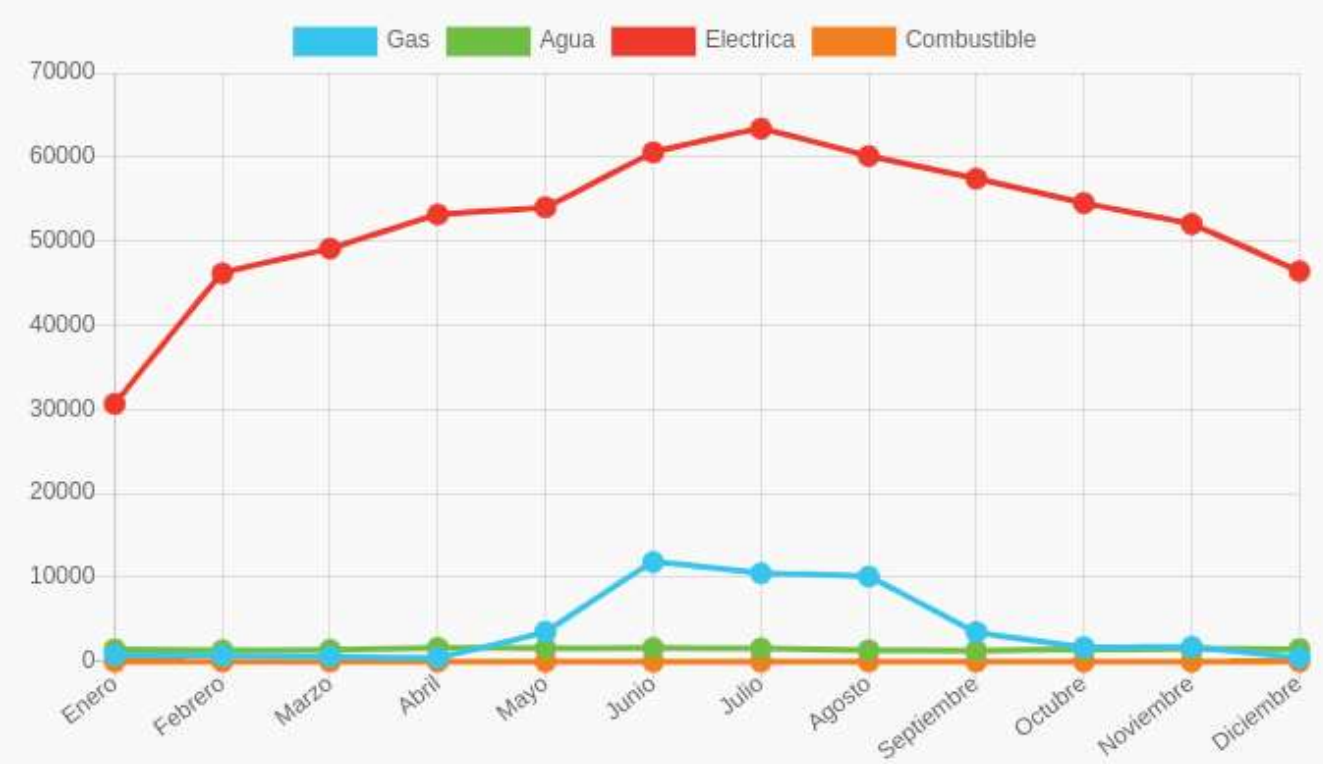

Figura 2: Consumo de energía.

Figure 2: Energy consumption 


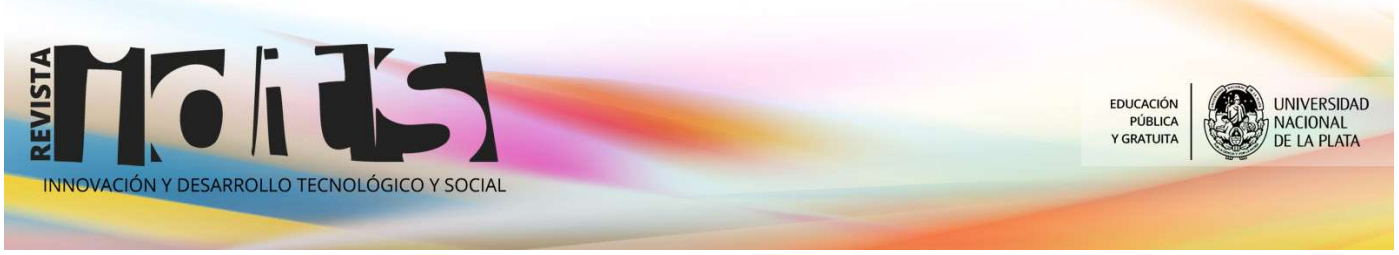

Innovación y Desarrollo Tecnológico y Social (2019) 1 (2): 28-35

Del llamado Relevamiento Energético de la PyME, el cual contiene el estudio de los consumos energéticos de cada proceso productivo, surge la necesidad de individualizarlos para el SGEfrlp y a cada una de estas partes del proceso productivo las llamamos "Unidades de servicio" y a los productos terminados los llamamos "Unidades de producción".

Para el estudio de los usos energéticos denominados en la Norma como USEs (usos significativos de energía), se conforman grupos de usos de energía para lo cual en el SGEfrlp se las llaman Líneas de producción de la empresa, ellas contienen las Unidades de servicio trabajando para obtener Unidades de producción o productos. Dicha Linea de producción se ve representada en la siguiente imagen [Figura 3].

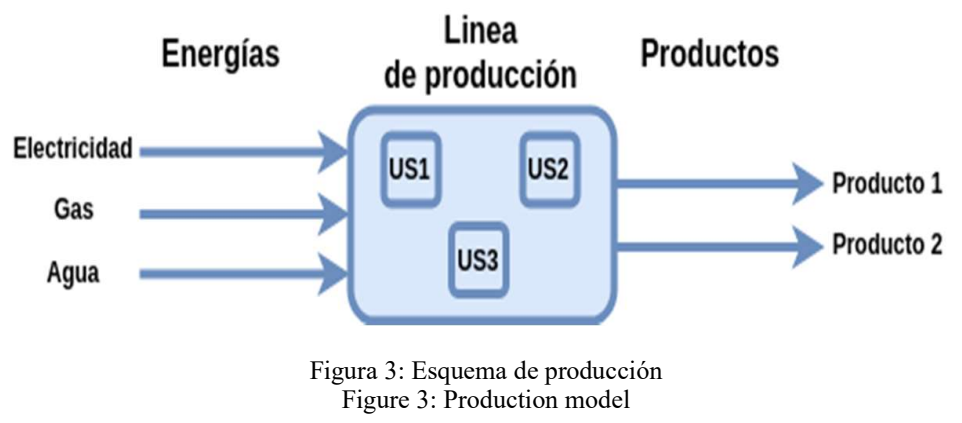

El SGEfrlp permite que se carguen mediciones del consumo de cada Unidad de servicio. En el caso de no contar con mediciones, permite que a través de datos tales como los tipos de energía que utiliza (electricidad, gas, agua o combustibles líquidos, otros), potencia nominal, rendimiento, estado de carga, velocidad real, ciclo de funcionamiento y la cantidad de horas que trabaja al año, obtener una energía equivalente por unidad de tiempo para cada Línea de Producción. De esta manera se puede conocer el peso que tiene, en el consumo total, cada Línea de Producción, la cual también se puede mostrar a través de una Matriz de Uso de Energía o mostrarlo a través de un gráfico tipo Torta [Figura 4].

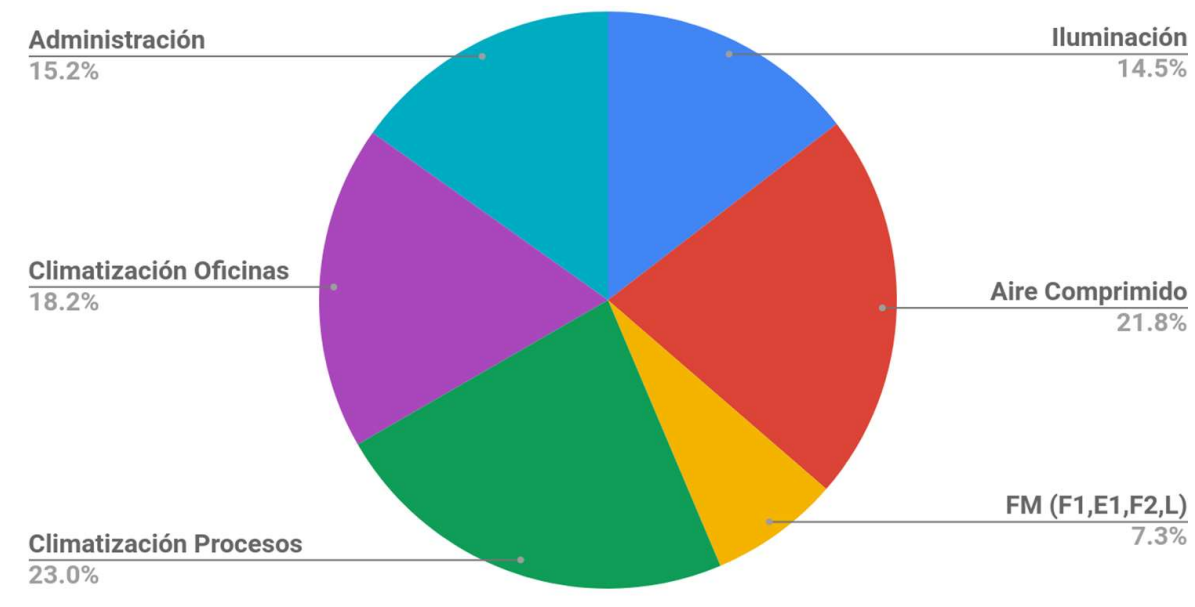

Figura 4: Matriz de Usos de Energía Eléctrica Figure 4: Electric power use matrix 


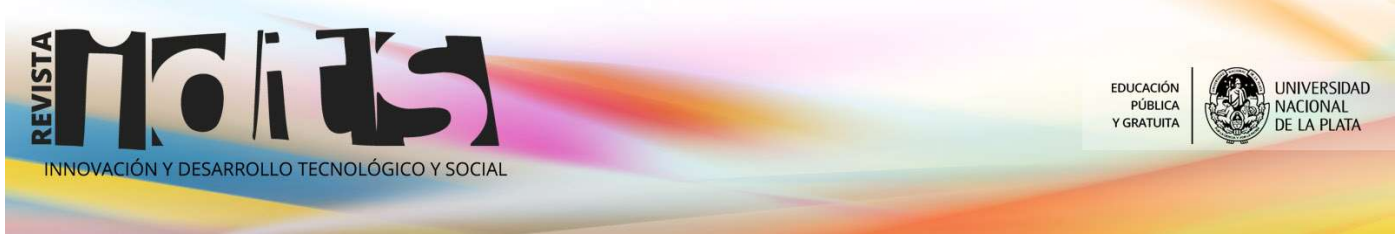

Innovación y Desarrollo Tecnológico y Social (2019) 1 (2): 28-35

Un punto de control nos lo da el hecho de que la sumatoria de los valores de energía utilizada (suma de usos de energía, llevados a la misma unidad, proveniente de la Matriz de Usos de Energía Total) debe ser aproximadamente igual a la sumatoria de energías consumida (suma de los consumos de energía, llevados a la misma unidad, proveniente de los datos de las facturas). Si este análisis se realiza mensualmente la matriz de consumos es la suma de las cantidades que figuran en las facturas de electricidad, gas, agua, combustible, otros, será aproximadamente igual a la Matriz de Usos de Energía Total de un mes de producción de la PyME.

De las Unidades de producción tenemos la cantidad de unidades que se producen por hora en cada Línea de producción, y sabiendo cuántas horas anuales trabaja dicha Línea sabemos cuántas Unidades de producción por periodo de tiempo se obtienen.

Uniendo la información de las Unidades de servicio, con la proveniente de las Unidades de producción, se obtiene el Indicador de cuánta energía de cada tipo contiene una Unidad de producción o producto. También se podría obtener la proyección de producción de la empresa y por ende la proyección de la energía que se consumirá para dicha producción.

El SGEfrlp le brinda a la empresa la posibilidad de trasladar rápidamente a los costos de producción el costo de las energías consumidas en su realización. Ya que cuenta con información del precio de la energía y de la cantidad de energía requerida para producir en tiempo real.

\section{Unidades de Producción por Día}

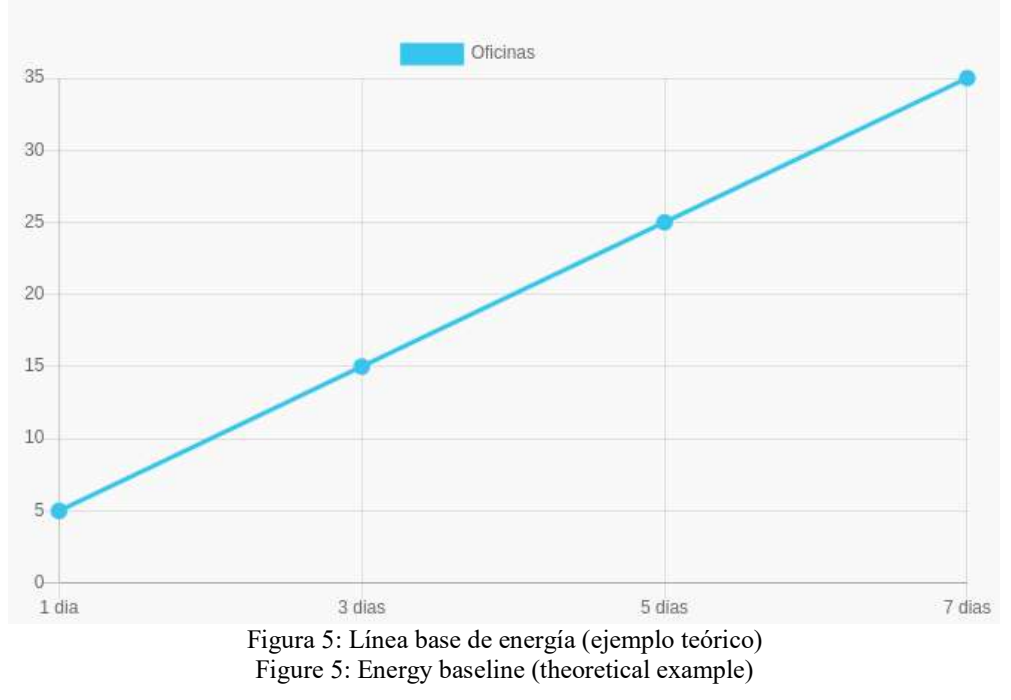

Sabiendo la cantidad de energía que consume la empresa para obtener una Unidad de producción es factible proponer una o varias Líneas Base de Energía (LBEs) [Figura 5], fotografía inicial o punto de partida que en conjunto con los Indicadores de Desempeño Energético (IDEs) permiten a la PyME realizar un seguimiento y control de los planes de acción tendientes a la mejora del Desempeño Energético.

En este punto del proceso la empresa conoce su Consumo temporal (historial de no menos de 12 meses), conoce como usa cada unidad de energía también en forma temporal (anual) y como impacta en la producción. Por lo tanto, la PyME está en condiciones de proponer objetivos y acciones tendientes a mejorar el Desempeño Energético (mayor eficiencia energética).

El SGEfrlp contempla que los Planes de Acción responda a una iniciativa concreta de mejora del desempeño energético, como por ejemplo el reemplazo de los Aires Acondicionados de baja eficiencia por los de tecnología Inverter; o pueden responder a las llamadas No Conformidades, 


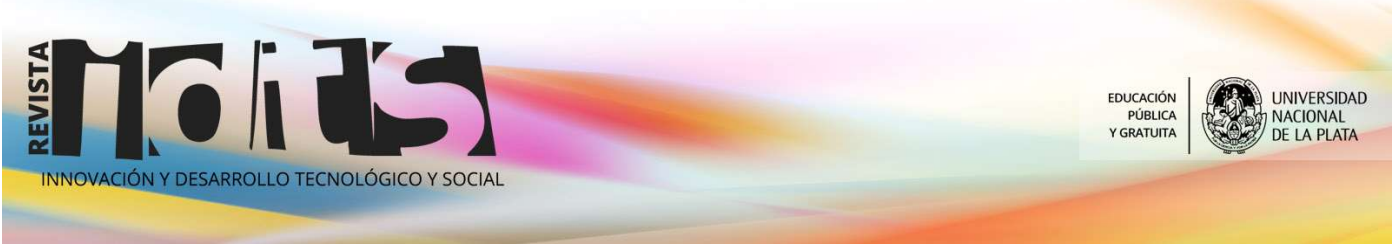

Innovación y Desarrollo Tecnológico y Social (2019) 1 (2): 28-35

así llamados los hallazgos provenientes de Auditorías Internas (realizadas por la propia empresa o un tercero) que indicaría desvíos con respecto a los requerimientos de la Norma.

Para el seguimiento y control de los Planes de Acción se cuenta con fecha estimada de realización, y un responsable de ejecución. Si no se pudiera cumplir en tiempo y forma, el SGEfrlp permite emitir una No Conformidad con el motivo por el cual no se pudo concretar el objetivo propuesto. Para lo cual el sistema cuenta con el motor necesario para que a través de datos se puedan obtener información valiosa para la toma de decisión y además provee la documentación de respaldo necesaria para la planificación, seguimiento y control de un Sistema de Gestión de Energía para una PyME.

Todo este camino en busca del objetivo general de la PyME de obtener una nueva Línea Base Energética cuya característica principal sea obtener la misma cantidad de Unidades de producción, con menos cantidad de Energía Consumida.

Es posible afirmar que los resultados que se obtengan en el presente proyecto permitirán clarificar la problemática de la gestión de la energía en las PyMEs, y de esta forma brindarles la posibilidad de un camino concreto a través del cual podrán generar incrementos reales en su competitividad en un entorno regional, nacional e internacional (RedTecNEE 2014).

En el año 2018 este desarrollo surge del trabajo de integración entre el CODAPLI (Codiseño Aplicado,y el grupo GyTE (Grupo y Tecnología Energética perteneciente los Departamentos de Ingeniería Eléctrica e Industrial), a su vez forma parte del Centro CODAPLI y de la RedTecNEE), tanto el CODAPLI como GyTE pertenecen a la Universidad Tecnológica Nacional Facultad Regional La Plata y aportan recursos humanos con perfiles específicos para llevar adelante este proyecto, por un lado especificando los requerimientos y necesidades del sistema y por el otro proveyendo diseño, programacion e implantacion del sistema.

Se plantea como trabajo futuro, mejorar el sistema producto de este proyecto, SGEfrlp, nutriendo la base de datos con medidores de energía que soporten tecnologías IoT, estos serían medidores que puedan acceder a nuestro sistema desde internet o desde la red de la empresa a través de una API para poder poblar las bases de datos con información medida y en tiempo real, así sabremos exactamente cuánta energía consume cada Unidad de servicio en cada momento pudiendo así hacer conjeturas y conclusiones sobre usos estacionales de la energía en forma online.

\section{$\underline{\text { Agradecimientos }}$}

El equipo de investigación agradece a la Prof. MariaInes Giuliano de la Vega por su valiosa contribución al realizar las traducciones de este trabajo al idioma inglés.

\section{Referencias bibliográficas}

RedTec, (2014) RED TECNOLOGICA NACIONAL SOBRE EFICIENCIA ENERGÉTICA, RedTecNEE - Presentado en CIDEL Argentina (2014)

Universidad Nacional de Colombia Facultad de Ingeniería y Administración Palmira, Colombia, (2016) Propuesta para la implementación de un sistema de gestión integral de la energía en la planta de Colombates S.A.

IRAM, (2018) ISO 50001 Sistemas de Gestión de la Energía - Requisitos con orientación para su uso. Disponible en: https://catalogo.iram.org.ar/\#/normas/detalles/11616 


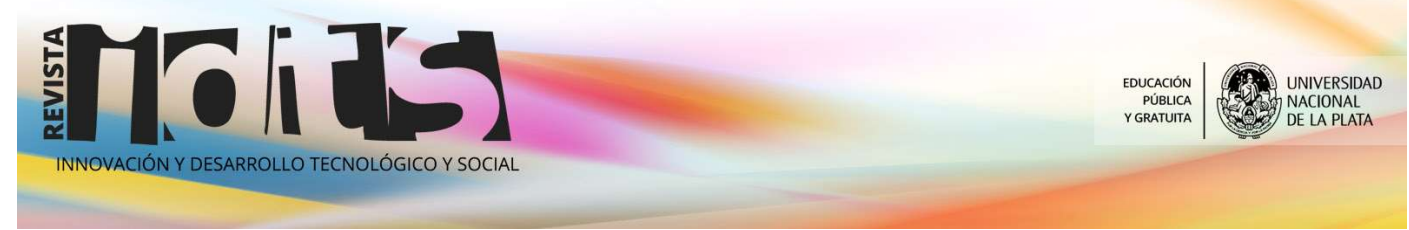

Innovación y Desarrollo Tecnológico y Social (2019) 1 (2): 28-35

AENOR, (2009) UNE 216501:2009 Auditorías energéticas. Requisitos. España. Disponible en: https://www.aenor.com/normas-y-libros/buscador-de-normas/UNE?c=N0043952

AENOR, (2007) UNE 216301:2007 Sistema de gestión energética. Requisitos. Disponible en https://www.aenor.com/normas-y-libros/buscador-de-normas/UNE?c=N0039927

Lyberg, Mats Douglas, (1987) Source Book for Energy Auditors - IEA energy conservation

Poder Ejecutivo Nacional, (2007) Decreto PEN 140/2007 - PRONUREE Programa Nacional de Uso Racional y eficiente de la Energía. Argentina 